\title{
The Effect of Surgical Resection on the Prognosis of Gastric Neuroendocrine Tumors
}

\author{
Halit Batuhan Demir* \\ Department of General Surgery, Ege University, Turkey \\ *Corresponding author: Halit Batuhan Demir, General Surgery Department, Ege University, Faculty of Medicine, Izmir, Turkey \\ To Cite This Article: Halit Batuhan Demir. The Effect of Surgical Resection on the Prognosis of Gastric Neuroendocrine Tumors. Am J Biomed \\ Sci \& Res. 2021 - 13(2). AJBSR.MS.ID.001848. DOI: 10.34297/AJBSR.2021.13.001848.
}

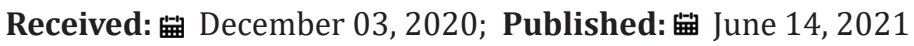

\section{Introduction}

Carcinoid tumors are known to cause several pathological pictures by the various peptides they produce, providing the continuity of disease. Previously, these tumors had been considered to be biologically and clinically more positively progressing tumors, unlike adenocarcinomas. However, upon the discovery of biological and clinical differences between pulmonary and intestinal carcinoids, these tumors have begun to be evaluated as a special type of a very different tumor family [1].

Gastric NETs (Neuroendocrine Tumors) are a type of tumor formed with excessive proliferation of enterochromaffin-like cells (ECL) that are especially localized in the gastric fundus and elevation of plasma gastrin levels, resulting in a series of neoplastic changes [2]. Multiple endocrine neoplasm (MEN-1) syndrome with the secretion of atrophic gastritis or gastrin related with low levels of acid may form the basis for hypergastrinaemia.

Pathophysiologically low acid level, hypergastrinaemia and increased ECL proliferation play a role in development of the disease. Evaluation of ECL cell proliferation, its histologic characterization, transformation and invasion degree has led to the development of strategies for the treatment of carcinoid tumors of the stomach [3].

In recent years, with the advancements of endoscopic techniques, the frequency of incidental detection of the disease has increased, while the advent of endoscopic resection improves on its treatment. Because of the low incidence of gastric NET in the general population, there is no established diagnosis and treatment algorithm even though there have been several clinical studies. Guidelines established by the WHO (World Health Organization) are currently used by numerous centers in the diagnosis, treatment and management of this disease.
Gastric NETs are among the rare tumors, accounting for $1 \%$ of all neuroendocrine tumors and less than $2 \%$ of all gastric tumors [4]. However, recent studies show an increased incidence of gastric carcinoid tumors.

It has been reported in a study examining three national databases that, the incidence of gastric NETs increased to $1.77 \%$ from $0.3 \%$ from 1950 s and increased to $8.7 \%$ from $2.4 \%$ among all carcinoid tumors [5].

A study using Surveillance, Epidemiology, and End Results (SEER) databases found that, the incidence of gastric NETs raised to $1.7 \%$ from $0.2 \%$ between 1973 and 1997 [6]. It was found in the same study that, $64 \%$ of patients with gastric NET detected were female patients and the incidence of non-carcinoid gastric cancer increased by two-fold in male patients. The reason of this increase in the incidence of gastric NET is not clear. This increase may suggest that gastric NETs are detected more frequently with upper gastrointestinal system endoscopy which has become more widespread, but the biological factors that may trigger the disease cannot be ignored. One of the proposed theories suggest that an increased use of acid-suppressing drugs may cause an increase in hypergastrinema induced carcinoid tumors. As the biology of these tumors are better understood, clinicians will provide more benefits in terms of the diagnosis, prevention and treatment of these disease.

Previously, gastric NETs had been divided into 3 types. However, a new type was recently added, Type- 4 Neuroendocrine Carcinoma. Gastric neuroendocrine carcinomas constitute a far and potentially uncorrelated subtype of less-differentiated, high-grade gastric carcinoids. In a series including such cases [7], 75\% of these tumors were developed by male patients and one third of all patients had 
hypergastrinaemia. Chronic atrophic gastritis (CAG) was found to be correlated with the incidence ( $82 \%$ ), while $25 \%$ of these patients showed ECL cell hyperplasia. These tumors are usually single at the time of diagnosis with a mean diameter greater than $4 \mathrm{~cm}$ and they either invaded or exceeded the muscularis propria. These rare tumors are most likely to give late manifestations; in a series, metastasis has been reported in all patients during diagnosis $[8,9]$. Aggressive cytoreductive surgery is extremely rarely indicated, but gastric resection may be required in order to resolve complications such as hemorrhage, obstruction or perforation.

\section{Prognosis}

Outcomes of patients with gastric carcinoid tumors are quite different and especially associated with the subtype of gastric lesion and with the underlying disease. Because the majority (>75\%) of gastric carcinoid tumors encountered in the clinical practice have type 1 lesions, outcomes are especially related to this type. Unfortunately, data collection by Godwin has led to the widespread recognition of the different types of gastric carcinoids. Since his study was conducted before the widespread use of endoscopy, it is necessary to make assessment in favor of more malignant lesions (defined only at surgery or autopsy). It is possible that Godwin's report might not take into account most of the relatively benign type 1 tumors. A more recent analysis by Modlin et al. [10] investigated 204 gastric carcinoid tumor cases obtained from about six thousand carcinoid tumor reports recorded in the SEER database between 1973 and 1991, and found 5-year survival rate as $48.6 \%$ for all gastric carcinoids.

5-year survival is $64 \%$ for localized lesions [11]. However, neither Godwin nor Modlin et al. [12] have recordings of individual carcinoid subtype for each patient. In a later study by Rappel and colleagues in 1995 on 110 patients with carcinoid tumor of the stomach, survival rate was found to be $78 \%$ and when corrected for age, this rate raised to $100 \%$ for 88 patients with type 1 lesion. Based on this data, Rappel concluded that patients with type 1 gastric carcinoid must not experience any change in their natural survival status. Although he outlines this situation as far from reality, in fact this well reflects that generally a benign prognosis should be expected for this subtype of gastric carcinoid tumors. Interestingly, in an update of SEER database by Modlin et al. [12], 5-year survival was considered as $63 \%$ for all gastric carcinoids and this rate was found as $69.1 \%$ for localized lesions. While a better prognosis is certainly more likely for type- 1 non-metastatic small carcinoids showing minimal invasion, the moderate rate in the latter study is somehow unexpected and suggests that these tumors may present more malignant behaviors than previously thought. Furthermore, possible morbidity and mortality that may occur during or after repeated endoscopic resections or removal of these lesions with antrectomy or gastrectomy should be considered. All conclusive discussions should include likelihood of later recurrence of these lesions and potential necessity of additional intervention. Normally life expectancy is optimistic in this patient group, but still there is an impression that the positive outcomes have been presented mostly for the majority of persons with type 1 lesions.

The incidence of metastatic spread related to gastric carcinoids in Zollinger-Ellison syndrome (ZES) is $10 \%$ and general prognosis of such gastric carcinoids is similar to type 1 gastric carcinoids [12]. In a recent case report, 38-year survival is reported in a patient with ZES, liver metastasis and gastric carcinoid who has been effectively treated with octreotide [13]. However, in general, longterm prognosis of type 2 disease ultimately reflects the course of MEN-1 gastrinoma (5-year survival rate: 60-75\%) [14]. The overall results table for sporadic gastric carcinoid tumors is poor compared to only ECL cell derived lesions or hypergastrinaemia. Five-year survival rate is directly associated with the degree of metastasis in less than $50 \%$ of such lesions [15-17].

\section{Objective}

The objective of this study is to perform a survival analysis in line with the WHO 2010 staging system in patients undergone surgical resection in our clinic with the diagnosis of Gastric NET and to investigate pathological factors affecting prognosis.

\section{Materials \& Methods}

The data of 27 patients who underwent surgical resection in our clinic due to the diagnosis of gastric NET between 2001 and 2015 were obtained from the information system of the hospital and screening of the file archives. Patients' demographics, clinical findings, type of operation, pathological outcome and overall survival were evaluated. Overall survival was screened via death notification system (DNS) and dates of death were identified. Based on the date of operation, overall survival time was calculated as months for patients who survived. Gastric NETs were classified as 4 types based on the epicrisis/discharge reports and pathologic reports of the patients. Tumor size, lymph node status, lymphovascular invasion and Ki-67 percentage were evaluated and their correlation with survival was investigated.

Gastric NET clinical typing was made as Type-1, Type-2 and Type-3. NEC was considered as Type-4. The types were determined using patients' serum gastrin levels, serum chromogranin levels and epicrisis clinical information. We have no patients with Type-2 gastric NET. In pathological outcomes, chromogranin, synaptophysin and neuron-specific enolase positivity were used as confirming factors in the determination of the types. Serum levels of chromogranin and gastrin were not used in statistical analysis.

Grouping based on the operational typing was made according to the notes of operation. Since laparoscopy or open surgery 
would not be compared, the operations were grouped only as total gastrectomy, subtotal gastrectomy and wedge resection. Tumor size, lymph node status, lymphovascular invasion and Ki-67 percentage were assessed according to pathologic outcomes.

Statistical analysis was performed using the SPSS 20.0 software package. The evaluation of categorical variables was performed using cross-tabulation analysis and Chi-square test. For numerical variables, comparisons of 2 groups were made with T-test in normally distributed data and with Mann-Whitney U-test in nonnormally distributed data. Whereas, comparison of more than 2 groups were carried out with ANOVA, and Bonferoni tests were used in numerical variables showing normal distribution and Kruskall Wallis tests for variables showing non-normal distribution. Statistical values are expressed as frequency, median, minimum, maximum and standard deviation. Statistical significance level was set at 0.05 .

\section{Results}

Out of 27 patients included, 14 (51.9\%) were male and 13 (48.1\%) female patients. Mean age of the patients was 55.8 and median age was 54 (33-81).

When complaints of the patients were examined, 12 (44.4\%) patients presented to the clinic with abdominal pain, 6 patients $(22.2 \%)$ with non-specific symptoms, 5 (18.5\%) patients with swallowing difficulty and $4(14.8 \%)$ patients with weakness.

Looking to the localization of gastric NETs, it was found that gastric NET was localized in the corpus in 10 (37\%) patients, in the fundus in $8(29.6 \%)$ patients, in the antrum in $5(18.5 \%)$ patients and in the cardia in $4(14,8 \%)$ patients.

When the patients were clinically and pathologically classified according to gastric NET classification, 15 patients (55.6\%) were found to have type 1, 5 (18.5\%) type 3 and 7 (25.9\%) type 4NEC disease. There were no patients found in the type 2 group associated with ZES and MEN 1.

According to the type of operation; 11 patients (40.7\%) had undergone total gastrectomy, 9 (33.3\%) subtotal gastrectomy (SG) and 7 (25.9\%) Wedge resection (WR). Hospitalization time was calculated in days and mean hospitalization was found as 18.48 days (6-35). There was a statistically significant correlation between type of operation and hospitalization time. Hospitalization time was shorter in patients undergone both subtotal gastrectomy and wedge resection compared to those patients undergone total gastrectomy. $(p=0.16)$.

Five (18.5\%) patients were exitus at follow-up and only one patient was lost at the postoperative first month due to additional comorbidities. The remaining 22 patients did not develop recurrence during follow-up. In the postoperative pathological report, a tumor was found to continue in the surgical margins in one patient who underwent subtotal gastrectomy. However, no macroscopic recurrence was observed in the control endoscopy.

According to the WHO 2010 guidelines, TNM staging results based on the pathologic outcomes are given in the table. Ten (37\%) patients were at stage 1, 7 (25.9) stage 2A, 2 (7.4\%) stage 2B, 7 stage $3 \mathrm{~B}$ and one patient was at stage 4 . None of our patients was at stage $3 \mathrm{~A}$.

Identifying information of the patients was obtained from the death notification system (DNS) and survival times were calculated in months. Patients who exited were found to be exitus at the months 1, 8, 25, 42 and 149. The mean follow-up duration was found as 57.8 months and median follow-up as 42 months (9-171) in 22 patients who were followed-up.

Looking to the distribution of patients who underwent surgical resection according to stages, we found that 9 of type 1 patients was at stage 1,2 were at stage $2 \mathrm{~A}$ and 4 at stage $3 \mathrm{~B}$. Whereas, of 5 type 3 patients, 3 were at stage $2 \mathrm{~A}$ and 2 at stage $2 \mathrm{~B}$. In Type 4 NEC patients group, one patient was at stage $2 \mathrm{~A}, 4$ were at stage $3 \mathrm{~B}$ and one at stage 4 .

As for the distribution of the type of operation according to the disease stages, out of 11 stage 1 patients, 5 underwent TG, 3 SG and $3 \mathrm{WR}$; one of 6 stage 2A patients underwent TG, 2 SG and 3 WR; one of stage $2 \mathrm{~B}$ patients underwent $\mathrm{TG}$ and the other patient underwent WR; out of 7 stage 3B patients, 4 underwent TG and 3 SG; while one patient at stage 4 underwent SG.

Since the number of patients was small and the distribution of the patients was not equal in the survival analysis according to stages; when stage 1 and $2 \mathrm{~A}$ tumors were compared as one noninvasive group, stage 2 as one group and stages $3 \mathrm{~B}$ and 4 one group in terms of survival, a statistically significant difference was found depending on the stages $(\mathrm{p}=0.045)$.

When tumor diameter was evaluated in millimeters ( $\mathrm{mm}$ ); the mean tumor diameter was found as $31 \mathrm{~mm}$ (2-220) and median tumor diameter as $20 \mathrm{~mm}$. (Chart-6) No significant difference was observed when tumor diameters were compared according to Ki67 and vascular invasion. Evaluating tumor diameter with survival, we found that mean tumor diameter was $5 \mathrm{~cm}$ in 5 patients who were exitus.

When Ki-67 values in the pathologic outcomes were evaluated in percentage, mean Ki-67 values was calculated as 16.6\% (1-80) and median Ki-67 as $4 \%$. Ki-67 cut-off value was found as $9.5 \%$ in our patients. No statistically significant difference was found between Ki-67 and the presence of vascular invasion. $(\mathrm{p}=0.147)$ When the correlation between Ki-67 and lymph node metastasis was evaluated, Ki-67 value was found to be higher in patients with lymph node metastasis ( $\mathrm{p}=0.021)$. 
It was found that one patient underwent Wedge resection, 2 patients underwent laparoscopic total gastrectomy and 4 patients underwent laparoscopic subtotal gastrectomy. Statistical evaluation was not carried out because of the small number of laparoscopic operations.

Out of 5 patients who were exitus, 4 had NEC and 1 Type 3 NET. Again, we found that 4 of these 5 patients had lymph node metastasis and all five patients pathologically had vascular invasion.

\section{Discussion}

Gastric NETs are rare tumors which typically show an inactive and slow growing course. However, all of them are potentially malignant and can metastasize to the liver independently from lymph node metastasis status. Five-year survival is 93\% in Type1 NETs, while this rate is reported as $50 \%$ for Type- 3 . When the biological behavior of tumor and survival were compared, currently recognized poor prognostic criteria include high grade of tumor, a tumor diameter greater than $3 \mathrm{~cm}$, mitoses above 9, vascular invasion and the presence of hepatic metastasis at the time of diagnosis. Because they are rarely encountered and different treatment algorithms are used in different centers, gastric NETs require a multidisciplinary approach.

In this study, no statistically significant difference was found related to prognosis in terms of tumor diameter, but we see that tumor diameters were greater than $3 \mathrm{~cm}$ in 5 patients who exited. Because of the lack of standardized evaluations in our clinic, the number of mitoses was not included in our study.

Although death is reported infrequently in patients with a tumor diameter under $2 \mathrm{~cm}$, recently total gastrectomy has been recommended in these patients. However, recent studies have demonstrated that endoscopic excision can be safely performed in the lesions smaller than $1 \mathrm{~cm}$ in type 1 and type 2 gastric NETs. Antrectomy can be used together with excision of the accessible lesions with a tumor diameter greater than $1 \mathrm{~cm}$ [18].

Similarly, to procedures reported in the literature, total gastrectomy, subtotal gastrectomy and local excision operations are also performed in our clinic. Besides one patient lost due to postoperative comorbidity, our 22 patients are still alive and disease free. Additionally, we see that tumor sizes were greater than $3 \mathrm{~cm}$ in 5 patients who exited. Again, one patient with positive lymph node who developed hepatic metastasis was exited at the 25th month of follow-up. Even when we consider the lack of information on cause of death and assume recurrence in all patients, 22 (82\%) of all patients survived during the 50-month follow-up period.

The TNM staging system of neuroendocrine tumors has not been adopted all over the world and although there are not many significant differences, disease staging can be made with different factors. The classification of carcinoids by WHO is recognized by American Joint Committee on Cancer (AJCC) and International Union Against Cancer (UICC) associations. Carcinoid term refers to well-differentiated benign lesions. Conversely, carcinoids are considered as high-grade lesions by European Neuroendocrine Tumor Society (ENETS). Both classifications can be used for the stomach [3].

Lymph node metastasis is reported as $5 \%$ for type $1,30 \%$ for type 2 and $71 \%$ for type 3 gastric NETs, while especially liver metastasis, distant organ metastasis is seen by $2.5 \%, 10 \%$ and $69 \%$, respectively [18]. In our study, rate of lymph node metastasis was found as $13 \%$ for Type $1,75 \%$ for Type 3 and $57 \%$ for NEC.

Type 1 and Type 2 NETs are often encountered as stage 1 or occasionally stage 2A. Whereas Type 3 and NEC (poorly differentiated endocrine carcinoma) is frequently seen as stages $2 \mathrm{~A}, 2 \mathrm{~B}$ and $3 \mathrm{~B}$, and rarely stage 4 (3). Confirming findings in the literature, our study also found that majority of Type 1 patients were at stages 1 and 2A, while type 3 and NEC patients were at stages $2 \mathrm{~B}, 3 \mathrm{~B}$ and 4 . We do not have any type 2 patients.

Prognosis may considerably vary in gastric NET patients. There are dramatic differences in prognosis between low-stage malignancies or those giving no symptoms and NEC or highstage malignancies. Good prognostic indicators include being confined to mucosa and submucosa, absence of vascular invasion, a tumor diameter $<1 \mathrm{~cm}$, absence of endocrine syndrome and being associated with CAG or MEN1-ZES. Most of type-1-CAG related gastric NETs have a perfect prognosis. Whereas NETs with aggressive behaviors have a poor prognosis because of invasion to the muscularis propria and beyond, tumor diameter being greater than $1 \mathrm{~cm}$, vascular invasion, presentation with endocrine syndrome, high mitotic activity and being sporadic [18]. In the present study, lack of gastric NET has been shown to be associated with MEN1 and ZES is a lacking aspect in terms of the evaluation of endocrine syndrome.

As the main source of gastrin in Type-1 gastric NETs, antrectomy together with excision of the lesion main source of gastrin provides healing by $80 \%$ (especially in a size greater than $1 \mathrm{~cm}$ ). Endoscopic excision can provide similar cure rates for lesions greater than $1 \mathrm{~cm}$, while there are studies reporting similar results achieved by longacting somatostatin analogs through control of hypergastrinaemia and ECL cell proliferation. In Type- 2 gastric NETS, first, excessive acid secretion and the formation of peptic ulcers are prevented with long-term PPI, while since there is not any effect on hypergastrinaemia, tumors may progress aggressively, requiring repeat surgery. Type- 3 gastric NETs of less than $1 \mathrm{~cm}$, progress with better outcomes with surgical treatment if there is an invasion to the lamina propria. Prognosis of NEC is generally poor. When 
presenting, most of NEC patients had invasion, advanced stage and short survival. Successful outcomes are achieved with aggressive surgery and adjuvant chemotherapy. Treatment may be determined in NECs, considering as adenocarcinomas of the stomach $[3,18]$. Similarly, in the present study we see that NEC patients have a poor prognosis with $57 \%$ mortality.

In a case series by Güler and Erikoğlu [19] in 2003, the results of 5 patients with Type-1 multiple gastric carcinoid between 1996 and 2001 were presented. The authors argued the necessity of total gastrectomy and lymph node dissection in multiple gastric carcinoids.

Until recently, total gastric resection has been recommended as the only surgical option for the treatment of gastric carcinoid tumors. In recent years, antrectomy which is a less aggressive method and proven to return elevated gastrin levels to normal has been recommended [20]. Wangberg [21] demonstrated that, even if hypergastrinaemia is reversed by removal of the antrum, enterochromaffin-like cell hyperplasia which occurs after a certain step is irreversible. In accordance with recent studies, in our study only 7 patients undergone Wedge resections maintained their disease-free survivals, showing that antrectomy may not be necessary.

Kımıloğlu et al. [22] evaluated tumor markers such as Ki-67, CD-117 and p53, and performed a survival analysis and found that the Ki-67 value was significant in prediction of prognosis. Similarly, in the present study we found that the prognosis of tumor was worse and the chance of lymph node metastasis was significantly different in high Ki-67 values.

Antrectomy should be performed at the hyperplastic stage especially before invasive carcinoid tumor is arisen. Gastrin levels return to normal only in half of gastric NET patients, while endoscopic follow-up continues in the other half. It is obvious that, a careful monitoring with gastroscopy and fundus biopsy will be necessary for as long as stomach tissue is left. Whereas, subtotal or total gastrectomy will be needed for locally invasive and metastatic tumors such as a wide primary lesion which presents nodular hyperplasia. The lesion should be excised in the cases of regional lymph node metastasis. Since sporadic NETs behave aggressively, their treatment is surgery. Lymph node dissection is recommended in addition to surgery if the tumor size is greater than $2 \mathrm{~cm} \mathrm{[16].}$ Unlike results found in the literature, in this study we achieved good survival outcomes only with WR in early-stage tumors.

\section{Conclusion}

In the present study, prognostic factors were examined in 27 patients with gastric NETs that are frequently encountered and significant results were obtained especially related to Ki-67. We presented what is done in our clinics regarding gastric NETs that have no sharp limits for treatment. In conclusion, according to the data of our clinic, a Ki-67 value which could be preoperatively assessed with an endoscopic biopsy above 9.5 may be a suggestion for lymph node dissection for gastric NETs.

The limiting factors of this study that may confound our statistical analysis include the limited number of patients, the fact that different parameters had been assessed by pathology over the many years from which patient data had been taken and the unevenly distributed stages and gastric NET types between patients.

\section{References}

1. Modlin I, Tang L (1996) The gastric enterochromaffin-like cell: an enigmatic cellular lesion. Gastroenterology 111(3): 783-810.

2. Gough D, Thompson G, Crotty T, Donohue JH, Kvols LK, et al. (1994) Diverse clinical and pathologic features of gastric carcinoid and the relevance of hypergastrinemia. World Journal of Surgery 18(4): 472480.

3. WHO (2010) Classification of Tumours. Pathology and Genetics of Tumours of the Digestive System. World Health Organization, Geneva, Switzerland, p. 64-68.

4. Godwin JD (1975) Carcinoid tumors-analysis of 2837 cases. Cancer 36(2): 560-569

5. Modlin IM, Lye KD, Kidd M (2004) A 50-year analysis of 562 gastric carcinoids: small tumor or larger problem? Am J Gastroenterol 99: 2332.

6. This reviews several national databases and demonstrates a clear increase in the incidence of gastric carcinoid tumors over a 50-year period.

7. Grep RO (1973) Histology. Mc Grow-Hill Book Company, London, UK.

8. Rindi G, Bordi C, Rappel S, La Rosa S, Stolte M, et al. (1996) Gastric carcinoids and neuroendocrine carcinomas: pathogenesis, pathology and behavior. World Journal of Surgery 20: 168-172.

9. Ahlman H, Kolby L, Lundell L, Olbe L, Wängberg B, et al. (1994) Clinical Management of gastric carcinoid tumors. Digestion 55: 77-85.

10. Modlin I, Sandor A, Tang L, Kidd M, Zelterman D (1997) A 40 year analysis of 265 gastric carcinoids. American Journal of Gastroenterology 92(4): 633-638.

11. Modlin I, Sandor A (1997) An analysis of 8,305 cases of carcinoid tumor Cancer 79: 813-829.

12. Modlin IM, Lye KD, Kidd M (2003) A 5-decade analysis of 13,715 carcinoid tumors. Cancer 97(4): 934-959.

13. Konturek SJ, Konturek PC, Bielanski W, Lorens K, Sito E, et al. (2002) Case presentation of gastrinoma combined withgastric carcinoid with th e longest survival record-Zollinger Ellison syndrome: pathophysiology, diagnosis and therapy. Medical Science Monitor 8(6): CS43-CS59.

14. Meko J, Norton J (1995) Management of patients with Zollinger - Ellison syndrome. Annual Review of Medicine 46: 395-411.

15. Hakanson R, Axelson J, Tielemens Y, Johansson AG, Willems G, et al (1992) Unilateral vagal denervation suppresses omeprazole- induced trophic effects on the denervated side of the rat stomach. Scand J Gastroenterol 27(1): 65-70. 
16. Rindi G, Azzoni C, La Rosa S, Klersy C, Paolotti D, et al. (1999) ECL cell tumor and poorly differentiated endocrine carcinoma of the stomach: prognostic evaluation by pathological analysis. Gastroenterology 116(3): 532-542

17. Tang L, Modlin I, Lawton G, Kidd M, Chinnery R (1996) The role of TGFalpha in the Enterochromaffin-like (ECL) cell tumor autonomy in an African Rodent Mastomys. Gastroenterology 111(5): 1212-1223.

18. Modlin IM, Lye KD, Kidd M (2004) A 50-year analysis of 562 gastric carcinoids: small tumor or larger problem? Am J Gastroenterol 99: 2332.

19. Güler A, Erikoğlu M (2003) Gastrik karsinoid tümörler. Genel Tıp Derg 13(2): 69-71.
20. Hirschowitz BI, Griffith J, Pelegrin D, Cummings OW (1992) Rapid regression of enterochromaffin like cell gastric carcinoids in pernicious anemia after antrectomy. Gastroenterol 102(4 Pt 1): 1409-1418.

21. Wangberg B, Nilsson O, Theodorsson E, Modlin IM, Dahlström A, et al. (1995) Are enterochromaffin like cell tumours reversible? An experimental study on gastric carcinoid induced in mastomys by histamine-2 receptor blockade. Regul Pept 56(1): 19-33.

22. Kımıloğlu SE, Erdoğan N, Ulusoy I, Samet E, Iğdem AA, et al. (2015) P53, KI-67, CD117 expression in gastrointestinal and pancreatic neuroendocrine tumours and evaluation of their correlation with clinicopathological and prognostic parameters. Turk J Gastroenterol 26(2): 104-111. 